\title{
HERMITIAN OPERATORS ON BANACH JORDAN ALGEBRAS
}

\author{
by M. A. YOUNGSON \\ (Received 16th December, 1977)
}

\section{Introduction}

In this note, we examine some of the properties of Hermitian operators on complex unital Banach Jordan algebras, that is, those operators with real numerical range. Recall that a unital Banach Jordan algebra $J$, is a (real or complex) Jordan algebra with product $a \circ b$, having a unit 1 , and a norm $\|\cdot\|$, such that $J$, with norm $\|\cdot\|$, is a Banach space, $\|1\|=1$, and, for all $a$ and $b$ in $J$,

$$
\|\boldsymbol{a} \circ \boldsymbol{b}\| \leqslant\|a\|\|b\| \text {. }
$$

We shall mainly be concerned with the Jordan analogues of $C^{*}$-algebras which were introduced by Wright in (13). These will be defined in Section 2, after which we shall state some of their properties. In Section 3, we prove a result on numerical ranges, which we require to complete a Vidav-Palmer theorem for complex unital Banach Jordan algebras, which was started in (15). Our final section is devoted to Hermitian operators on the Jordan analogue of $C^{*}$-algebras. Using a generalisation of results of Sinclair for $C^{*}$-algebras (10), we show that an operator on such a Jordan algebra $A$ is Hermitian if and only if it satisfies an algebraic equation involving only the elements of $A$. This characterisation was also obtained by Kaup (8) using deep results connecting Jordan triple systems and symmetric complex Banach manifolds. Finally, as an application, we give a large class of Hermitian operators, whose squares are not Hermitian.

We shall use the following notation:

(a) If $X$ is a normed linear space, we denote by $X^{\prime}$, the dual of $X$ and by $B(X)$, the bounded linear operators on $X$. If $S$ is a non-empty subset of $X$, and $r \in R$, we let $r S=\{r s: S \in S\}$ and, co $S$ and co $S$ respectively the convex hull of $S$ and the closed convex hull of $S$.

(b) If $J$ is a Banach Jordan algebra, we let

$$
\{a, b, c\}=(a \circ b) \circ c-(c \circ a) \circ b+(b \circ c) \circ a
$$

for all $a, b$ and $c$ in $J$. For $a$ in $J$, we define $L_{a}$ and $U_{a}$ in $B(J)$ by

$$
L_{a}(x)=a \circ x \text { and } U_{a}(x)=\{a, x, a\}
$$

for all $x$ in $J$. Then by (6) p. 36, equation 60 , for all $a$ in $J$,

$$
\left(L_{a}\right)^{2}=\frac{1}{2}\left(L_{a^{2}}+U_{a}\right)
$$


If $J$ is special, that is, $J$ is also a subset of an associative algebra, and $x \circ y=$ $\frac{1}{2}(x y+y x)$, where $x y$ is the associative product, then, for all $a, b$ and $c$ in $J$,

$$
\{a, b, c\}=\frac{1}{2}(a b c+c b a) \text {. }
$$

(c) If $S$ is a non-empty subset of a complex unital Banach Jordan algebra $J$, let

$$
E(S)=\overline{\operatorname{co}}\{\exp (i s): s \in S\} \text {. }
$$

Note that, for each $a \in J$, the closure of the Jordan algebra generated by 1 and $a$ is a complex unital Banach algebra, as multiplication is continuous. In particular, for all $a$ in $J, \exp (i a)$ is well defined.

\section{2. $J B^{*}$-algebras and $J B$-algebras}

A $J B^{*}$-algebra is a complex, unital Banach Jordan algebra $A$, with an involution * such that, for all $x$ in $A$,

$$
\left\|\left\{x, x^{*}, x\right\}\right\|=\|x\|^{3} .
$$

In his definition of Jordan $C^{*}$-algebras in (13), Wright has the additional hypothesis that, for all $x$ in $A$,

$$
\|x\|=\|x *\|
$$

However, in (15), we showed that this condition is automatically satisfied in a $J B^{*}$-algebra, so that the two classes coincide.

As the name suggests, $J B^{*}$-algebras are closely linked to the $J B$-algebras introduced by Alfsen, Shultz and Størmer in (1). A JB-algebra is a real, unital, Banach Jordan algebra $K$, such that, for all $a$ and $b$ in $K$,

$$
\left\|a^{2}+b^{2}\right\| \geqslant\left\|a^{2}\right\|=\|a\|^{2} \text {. }
$$

The main result of (13) shows that the self-adjoint elements of a $J B^{*}$-algebra form a $J B$-algebra, while, conversely, a $J B$-algebra may be embedded as the self-adjoint elements of a $J B^{*}$-algebra.

Examples of $J B$-algebras include any norm-closed real Jordan subalgebra $K$ of self-adjoint operators on a Hilbert space such that $1 \in K$; these are called $J C$-algebras in (11), and $M_{3}^{8}$, the single, formally real, simple, non-special, finite dimensional Jordan algebra (see (1), (6) and (7)). Hence not all $J B$-algebras are special, and, in particular, not all $J B$-algebras are isometrically isomorphic to $J C$-algebras. However, from Corollary 5.7 and Theorems 8.6 and 9.5 of (1), we obtain the following result.

Theorem 1. If $A$ is a JB-algebra, there exists a family $G$ of continuous homomorphisms $\phi: A \rightarrow B_{\phi}$ such that

(a) for each non-zero $a$ in $A$, there is $\phi \in G$ such that $\phi(a) \neq 0$ and

(b) for each $\phi \in G$, either $B_{\phi}=M_{3}^{8}$ or $B_{\phi}$ is a JC-algebra.

This was used to prove the following Theorem and Corollary in (13). 
Theorem 2. If $A$ is $a J B$-algebra, and $a$ and $b$ are elements of $A$, then the closure of the Jordan algebra generated by $1, a$ and $b$ is a JB-algebra, isometrically isomorphic to a JC-algebra.

Corollary 3. If $A$ is $a J^{*}$-algebra, and $a$ and $b$ are self-adjoint elements of $A$, then the closure of the complex Jordan algebra generated by $1, a$, and $b$ is $a$ $J B^{*}$-algebra, (with involution inherited from $A$ ) which is ${ }^{*}$-isometrically isomorphic to a self-adjoint norm-closed complex Jordan subalgebra $J$ of operators on a Hilbert space, such that $1 \in J$; these are called JC*-algebras in (2).

In particular, by choosing $a=b$ in Theorem 2 and Corollary 3, we may obtain the following Corollary. A proof not depending on Theorem 1 can be found in (1) Proposition 2.3 and (13), p. 292.

Corollary 4. (a) If $A$ is a $J B$-algebra and $a \in A$, then the closure of the algebra generated by 1 and $a$ is a JB-algebra, isometrically isomorphic to the self-adjoint part of a commutative $C^{*}$-algebra with identity.

(b) If $A$ is a $\mathrm{JB}^{*}$-algebra, and $a$ is a self-adjoint element of $A$, then the closure of the complex algebra generated by 1 and a is a $J B^{*}$-algebra, *-isometrically isomorphic to a commutative $C^{*}$-algebra with identity.

We conclude this section with a characterisation of the centre of a $J B^{*}$-algebra. Recall that if $J$ is a Banach Jordan algebra, and $a, b \in J$, then $a$ and $b$ operator commute if $L_{a}$ commutes with $L_{b}$ in $B(J)$. (See (6) and (7)). The following Theorem was obtained in the $J C^{*}$-algebra case by Topping (12).

Theorem 5. Let A be a JB*-algebra and a be a self-adjoint element of $A$. Then the following are equivalent:

(i) a operator commutes with all self-adjoint elements of $A$.

(ii) $a^{2} \circ b=\{a, b, a\}$ for all self-adjoint elements $b$ of $A$.

(iii) If $J$ is any closed, complex, self-adjoint Jordan subalgebra of $A$ such that $a \in J$, and if $F: J \rightarrow B(H)$ is any continuous Jordan *-homomorphism, then, for all self-adjoint elements $b$ of $J$,

$$
F(a) F(b)=F(b) F(a)
$$

Proof. (a) (i) $\Rightarrow$ (ii). Let $b$ be a self-adjoint element of $A$. Then, for all $c$ in $A$,

$$
a \circ(b \circ c)=b \circ(a \circ c) \text {. }
$$

In particular, putting $a=c$, we have $a \circ(a \circ b)=a^{2} \circ b$, so

$$
\left(L_{a}\right)^{2}(b)=L_{a^{2}}(b) \text {. }
$$

But $\left(L_{a}\right)^{2}=\frac{1}{2}\left(L_{a^{2}}+U_{a}\right)$, so $L_{a^{2}}(b)=U_{a}(b)$, or

$$
a^{2} \circ b=\{a, b, a\} .
$$

(b) (ii) $\Rightarrow$ (iii). Let $J$ be any closed, complex, self-adjoint Jordan subalgebra of $A$, 
such that $a \in J$, and let $F: J \rightarrow B(H)$ be any continuous Jordan *-homomorphism. Let $b \in J$ be self-adjoint and let $c=F(a)$ and $d=F(b)$. Then $c$ and $d$ are self-adjoint and

$$
c^{2} \circ d=\{c, d, c\} \text {. }
$$

Hence $\frac{1}{2}\left(c^{2} d+d c^{2}\right)=c d c$, so

$$
c(c d-d c)=(c d-d c) c .
$$

By the Kleinecke-Shirokov theorem, see for example (3) Proposition 18.13, $c d-d c$ is quasinilpotent. But $c$ and $d$ are self-adjoint, so $i(c d-d c)$ is a self-adjoint quasinilpotent, and hence is zero, as required.

(c) (iii) $\Rightarrow$ (ii). Let $b \in A$ be self-adjoint. By Corollary 3 , the closure of the complex Jordan algebra generated by $1, a$ and $b$ is isometrically *-isomorphic to a $J C^{*}$-algebra. Denoting this *-isomorphism by $\pi$, we have, by hypothesis, $\pi(a) \pi(b)=$ $\pi(b) \pi(a)$, and so

and

$$
\begin{aligned}
& \pi\left(a^{2}\right) \circ \pi(b)=\{\pi(a), \pi(b), \pi(a)\} \\
& \pi\left(b^{2}\right) \circ \pi(a)=\{\pi(b), \pi(a), \pi(b)\} .
\end{aligned}
$$

As $\pi^{-1}$ is a Jordan isomorphism

and

$$
\begin{aligned}
& a^{2} \circ b=\{a, b, a\} \\
& b^{2} \circ a=\{b, a, b\} .
\end{aligned}
$$

(d) (iii) $\Rightarrow$ (i). Let $b$ be a self-adjoint element of $A$, and suppose that $L_{a}$ does not commute with $L_{b}$. Then there exists $c$ in $A$ such that

$$
a \circ(b \circ c) \neq b \circ(a \circ c) \text {. }
$$

By replacing $c$ with $\frac{1}{2}\left(c+c^{*}\right)$ or $\frac{1}{2} i\left(c^{*}-c\right)$ if necessary, we may assume that $c$ is self-adjoint.

By Theorem 1, there is a continuous Jordan homomorphism $\phi$, from the self-adjoint elements of $A$ to $B_{\phi}$, such that

$$
\phi(a) \circ(\phi(b) \circ \phi(c)) \neq \phi(b) \circ(\phi(a) \circ \phi(c))
$$

and either $B_{\phi}$ is a $J C$-algebra, or $B_{\phi}$ is $M_{3}^{\mathbf{8}}$.

If $B_{\phi}$ is a $J C$-algebra, then $\phi(a) \phi(b)=\phi(b) \phi(a)$ by hypothesis, and so

$$
\begin{aligned}
\phi(a) \circ(\phi(b) \circ \phi(c)) & =\frac{1}{4}(\phi(a)(\phi(b) \phi(c)+\phi(c) \phi(b))+(\phi(b) \phi(c)+\phi(c) \phi(b)) \phi(a)) \\
& =\frac{1}{4}(\phi(b) \phi(a) \phi(c)+\phi(a) \phi(c) \phi(b)+\phi(b) \phi(c) \phi(a)+\phi(c) \phi(a) \phi(b)) \\
& =\phi(b) \circ(\phi(a) \circ \phi(c))
\end{aligned}
$$

which is a contradiction.

If $B_{\phi}$ is $M_{3}^{8}$, let $p$ be any idempotent in $B_{\phi}$ and $d$ be any self-adjoint element of $A$ such that $\phi(d)=p$. From part $(c)$,

$$
d^{2} \circ a=\{d, a, d\}
$$

so

$$
p^{2} \circ \phi(a)=\{p, \phi(a), p\}
$$


and hence

$$
L_{p}(\phi(a))=U_{p}(\phi(a)) .
$$

By (1), Lemma $2.11, \phi(a)$ operator commutes with $p$, so in particular,

$$
(\phi(a) \circ \phi(c)) \circ p=(p \circ \phi(c)) \circ \phi(a) .
$$

Now, as $M_{3}^{8}$ is a finite dimensional $J B$-algebra, every element of $M_{3}^{8}$ is a finite linear combination of idempotents, by (1) Proposition 4.3. Thus

$$
(\phi(a) \circ \phi(c)) \circ \phi(b)=(\phi(b) \circ \phi(c)) \circ \phi(a) .
$$

This is also a contradiction, and hence $a$ must operator commute with $b$.

Remark. The last part of the above proof works in any $J B$-algebra with "sufficiently many" idempotents.

If $A$ is a $J B^{*}$-algebra, let $C(A)$ denote the self-adjoint elements of $A$ which operator commute with all self-adjoint elements of $A$. The centre of $A$ is $C(A)+$ $i C(A)$, and it can be shown to be a $B^{*}$-algebra. In the case that $A$ is a $B^{*}$-algebra, it follows from Theorem 5 that this definition of centre agrees with the usual one.

\section{The Numerical Range}

If $A$ is a complex unital Banach Jordan algebra, let $D(A, 1)$ denote the normalised states on $A$, that is

$$
D(A, 1)=\left\{f \in A^{\prime}: f(1)=\|f\|=1\right\} .
$$

Given $a \in A$, the numerical range $V(A, a)$ is defined by

$$
V(A, a)=\{f(a): f \in D(A, 1)\}
$$

The Hermitian elements of $A$, denoted by Her $A$, are those elements of $A$ with real numerical range.

Since, for each $a$ in $A$, the closure of the Jordan algebra generated by 1 and $a$ is a commutative unital Banach algebra, many of the well known results on the numerical range of elements of Banach algebras go through without change to Banach Jordan algebras, see (15). Here, we wish to show that $V(A, a)=V\left(B(A), L_{a}\right.$ ) (where $V\left(B(A), L_{a}\right)$ is the numerical range of the operator $L_{a}$ in the Banach algebra $\left.B(A)\right)$. First, we recall that the spatial numerical range $W\left(L_{a}\right)$ is defined by

$$
W\left(L_{a}\right)=\left\{f(a \circ x): f \in A^{\prime}, x \in A \text { and } f(x)=\|x\|=\|f\|=1\right\},
$$

and that from normed linear space theory (see (4) Theorem 9.4), for all $a$ in $A$,

$$
\overline{\operatorname{co}} W\left(L_{a}\right)=V\left(B(A), L_{a}\right) \text {. }
$$

Theorem 6. If $A$ is a complex unital Banach Jordan algebra, and $a \in A$, then

$$
V(A, a)=V\left(B(A), L_{a}\right)=W\left(L_{a}\right) .
$$

Hence $a \in \operatorname{Her} A$ if and only if $L_{a} \in \operatorname{Her} B(A)$. 
Proof. If $f \in D(A, 1)$, then $\|f\|=f(1)=\|1\|=1$, and so, for $a \in A, f(a)=f(a \circ 1) \in$ $W\left(L_{a}\right)$. Hence for all $a$ in $A, V(A, a) \subseteq W\left(L_{a}\right)$.

Conversely, if $x \in A$ and $f \in A^{\prime}$ are such that $\|f\|=\|x\|=f(x)=1$, define a linear functional $F$ on $A$ by $F(b)=f(b \circ x)$ for $b$ in $A$. Clearly $F(1)=1$, while for all $b$ in $A$, $\mid F(b) \leqslant\|f\| b \circ x\|b\|$. Hence $F \in D(A, 1)$, and so for all $a$ in $A, W\left(L_{a}\right) \subseteq V(A, a)$.

Thus $V(A, a)=W\left(L_{a}\right)$. By (15) Theorem $2(i), V(A, a)$ is closed and convex. So

$$
W\left(L_{a}\right)=V(A, a)=\overline{\operatorname{co}} V(A, a)=\overline{\operatorname{co}} W\left(L_{a}\right)=V\left(B(A), L_{a}\right) .
$$

Remark. Theorem 6 also holds if $A$ is only a (non-associative) algebra, with unit, which is a Banach space under a norm, which is submultiplicative with respect to the product, and such that the norm of the unit is one.

\section{The Vidav-Palmer Theorem for Banach Jordan algebras}

In (15), we showed that if $A$ is a $J B^{*}$-algebra, then $A=\operatorname{Her} A \oplus i \operatorname{Her} A$, and Her $A$ is the real Jordan algebra of self-adjoint elements of $A$. Conversely, we showed that if $A$ is a complex unital Banach Jordan algebra such that $\operatorname{Her} A$ is a real Jordan algebra and $A=\operatorname{Her} A \oplus i$ Her $A$, then there is a continuous involution ${ }^{*}$ on $A$, called the natural involution on $A$, defined by

$$
(h+i k)^{*}=h-i k
$$

for $h$ and $k$ in $A$, and an equivalent norm on $A$, such that $A$, with the natural involution is a $J B^{*}$-algebra in the new norm. In Theorem 8 , we shall prove that the new norm is in fact equal to the original norm. First, we require a Lemma.

Lemma 7. If $K$ is a complex Banach algebra with a unit, and $J$ a closed complex Jordan subalgebra of $K$, containing the unit, then, for all $a$ and $b$ in $J$,

$$
\{\exp (i a), b, \exp (i a)\}=\left(\exp 2 i L_{a}\right)(b) .
$$

Proof. Let $a \in J$. By writing down the power series expansion for exp $2 i L_{a}$, it is clear that for $b$ in $J$, $\left(\exp 2 i L_{a}\right)(b)$ does not depend on whether we regard $L_{a}$ as an element of $B(J)$ or $B(K)$. Thus, for the remainder of the proof, we regard $L_{a}$ as an element of $B(K)$.

Put $h=i a$, and define $l_{h}$ and $r_{h} \in B(K)$, by

$$
l_{h}(x)=h x \text { and } r_{h}(x)=x h,
$$

for $x$ in $K$. Then as $2 i L_{a}=l_{h}+r_{h}$, and $l_{h}$ commutes with $r_{h}$ we have, for all $b$ in $K$,

$$
\begin{aligned}
\left(\exp 2 i L_{a}\right)(b) & =\left(\exp \left(l_{h}+r_{h}\right)\right)(b) \\
& =\exp l_{h} \exp r_{h}(b) \\
& =(\exp h)(b)(\exp h) \\
& =\{\exp (i a), b, \exp (i a)\} .
\end{aligned}
$$

In particular, the required result holds for all $b$ in $J$. 
Theorem 8. Let $A$ be a complex unital Banach Jordan algebra such that Her $A$ is $a$ real Jordan algebra and $A=\operatorname{Her} A \oplus i \operatorname{Her} A$. Then $A$ with its natural involution is a $J B^{*}$-algebra.

Proof. Fix $y \in A$. By Corollary 3, and (15) Theorem 11, there is a homeomorphic *-isomorphism $\pi$ from $Q$, the closure of the complex Jordan algebra generated by 1 , $\frac{1}{2}\left(y+y^{*}\right)$ and $\frac{1}{2} i\left(y-y^{*}\right)$ to a $J C^{*}$-algebra. Further by (15), Lemma 12

$$
\|x\| \leqslant\|\pi(x)\|
$$

for all $x$ in $Q$.

Let $h \in$ Her $Q$. As $\pi$ is a homeomorphic *-isomorphism, for all $x$ in $Q$, by Lemma 7, $\{\exp (i h), x, \exp (i h)\}=\left(\exp \left(2 i L_{h}\right)\right)(x)$. As $h \in \operatorname{Her} Q, L_{h} \in \operatorname{Her} B(Q)$ by Theorem 6, so that by (4) Lemma 5.2,

$$
\left\|\exp \left(2 i L_{h}\right)(x)\right\|=\|x\|
$$

for all $x$ in $Q$. Thus, for all $x$ in $Q$,

$$
\|\{\exp (i h), x, \exp (i h)\}\|=\|x\| .
$$

The identity $\left\{v,\left\{w, v^{2}, w\right\}, v\right\}=\{v, w, v\}^{2}$ is valid in any special Jordan algebra and hence, by Macdonald's Theorem (see (6), Section 1.9) in every Jordan algebra. Thus for $h \in \operatorname{Her} Q$ and $x \in Q$, we have

$$
\begin{aligned}
\|\{x, \exp i h, x\}\| & =\left\|\left\{\exp \left(\frac{1}{2} i h\right),\{x, \exp i h, x\}, \exp \left(\frac{1}{2} i h\right)\right\}\right\| \\
& =\left\|\left\{\exp \left(\frac{1}{2} i h\right), x, \exp \left(\frac{1}{2} i h\right)\right\}^{2}\right\| \\
& \leqslant\left\|\left\{\exp \left(\frac{1}{2} i h\right), x, \exp \left(\frac{1}{2} i h\right)\right\}\right\|^{2} \\
& =\|x\|^{2},
\end{aligned}
$$

using (2) twice. Now let $\kappa, \eta>0$. Pick $a \in Q$ such that $\|a\|=1$ and $\|\pi(a)\|>\|\pi\|-\eta$. Then $\left.\left\|\pi\left(a^{*}\right)\right\|=\| \pi(a)\right)^{*}\|=\| \pi(a) \|$, so that,

$$
\pi\left(a^{*}\right) \in\|\pi(a)\| E(\pi(\operatorname{Her} Q))
$$

using (15) Theorem 8. As $\pi$ is a homeomorphic *-isomorphism, this implies

$$
a^{*} \in\|\pi(a)\| E(\operatorname{Her} Q) \text {. }
$$

Hence let $z \in\|\pi(a)\| \operatorname{co}\{\exp i h: h \in \operatorname{Her} Q\}$ be such that $\left\|a^{*}-z\right\|<\kappa$. So $z=$ $\sum_{i=1}^{n} b_{j} \exp \left(i h_{j}\right)$ where $h_{j} \in \operatorname{Her} Q, b_{j} \in R$, with $b_{j} \geqslant 0$ for all $j$ and $\sum_{j=1}^{n} b_{j} \leqslant\|\pi(a)\|$. Then

$$
\begin{aligned}
\|\{a, z, a\}\| & \leqslant \sum_{j=1}^{n} b_{j}\left\|\left\{a, \exp \left(i h_{j}\right), a\right\}\right\| \\
& \leqslant \sum_{i=1}^{n} b_{j}\|a\|^{2} \\
& \leqslant\|\pi(a)\| \\
& \leqslant\|\pi\| .
\end{aligned}
$$


As $\left\|\left\{a, a^{*}, a\right\}\right\| \leqslant\|\{a, z, a\}\|+\left\|U_{a}\right\| \kappa$,

$$
\begin{aligned}
\|\pi(a)\|^{3}=\left\|\pi\left(\left\{a, a^{*}, a\right\}\right)\right\| & \leqslant\|\pi\|\left\|\left\{a, a^{*}, a\right\}\right\| \\
& \leqslant\|\pi\|^{2}+\|\pi\|\left\|U_{a}\right\| \kappa .
\end{aligned}
$$

As this holds for all $\kappa>0,\|\pi(a)\|^{3} \leqslant\|\pi\|^{2}$. Thus

$$
(\|\pi\|-\eta)^{3} \leqslant\|\pi\|^{2} \text {. }
$$

As this holds for all $\eta>0,\|\pi\|^{3} \leqslant\|\pi\|^{2}$, so $\|\pi\| \leqslant 1$. Combining this with (1), we conclude that $\pi$ is an isometry. Hence

$$
\left\|\left\{y, y^{*}, y\right\}\right\|=\left\|\pi\left(\left\{y, y^{*}, y\right\}\right)\right\|=\|\pi(y)\|^{3}=\|y\|^{3} .
$$

Thus $A$ is a $J B^{*}$-algebra.

\section{Hermitian Operators on $J^{*}$-algebras}

In this section, we extend results of Sinclair (10) concerning Hermitian operators on a $C^{*}$-algebra with identity. First we recall that a derivation $\delta$ on a $J B^{*}$-algebra $A$ is a linear map $\delta: A \rightarrow A$ such that, for all $x$ and $y$ in $A$,

$$
\delta(x \circ y)=\delta(x) \circ y+x \circ \delta(y)
$$

and a *-derivation on $A$ is a derivation $\delta$ on $A$ such that, for all $x$ in $A$,

$$
\delta\left(x^{*}\right)=-(\delta(x))^{*}
$$

It is routine to check that if $\delta$ is any derivation on a $J B^{*}$-algebra, then $\delta(1)=0$, and that $\delta$ may be decomposed as a sum of two *-derivations. Also, by modifying the usual argument for $C^{*}$-algebras, see for example (9) Lemma 4.1.3, it can be shown that every derivation on a $J B^{*}$-algebra is continuous.

We have shown that, if $A$ is a $J B^{*}$-algebra, and $a \in \operatorname{Her} A$, then $L_{a} \in \operatorname{Her} B(A)$. In this section we shall show that every ${ }^{*}$-derivation on $A$ is a Hermitian operator on $A$, and conversely that every Hermitian operator on $A$ is of the form $L_{a}+\delta$ where $a \in \operatorname{Her} A$ and $\delta$ is a *-derivation.

We start with some routine properties of derivations. Parts (i), (ii) and (iii) of the following Theorem are analogues of the Banach algebra case, and (ii) and (iii) are shown for finite dimensional Jordan algebras in (5). For this reason, we shall only prove (iv).

Theorem 9. Let $A$ be a JB*-algebra and $\delta a{ }^{*}$-derivation on $A$.

$$
\delta^{m}(u \circ v)=\sum_{k=0}^{m}\left(\begin{array}{c}
m \\
k
\end{array}\right)\left(\delta^{k} u\right) \circ\left(\delta^{m-k} v\right)
$$

for all $u, v$ in $A$, and all positive integers $m$.

(ii) $\exp ($ it $\delta)$ is $a^{*}$-automorphism for all $t \in \mathbf{R}$.

(iii) Conversely, if $T \in B(A)$ is such that $\exp (i t T)$ is a *automorphism for all $t \in \mathbf{R}$, then $T$ is $a^{*}$-derivation.

(iv) $\delta\left\{b, c^{*}, d\right\}=\left\{\delta b, c^{*}, d\right\}-\left\{b,(\delta c)^{*}, d\right\}+\left\{b, c^{*}, \delta d\right\}$ for all $b, c$ and $d$ in $A$. 
Proof of (iv).

$$
\begin{aligned}
\delta\left\{b, c^{*}, d\right\}= & \delta\left(\left(b \circ c^{*}\right) \circ d-(b \circ d) \circ c^{*}+\left(c^{*} \circ d\right) \circ b\right) \\
= & \left(b \circ c^{*}\right) \circ \delta d+\left(\delta b \circ c^{*}\right) \circ d-\left(b \circ(\delta c)^{*}\right) \circ d \\
& +(b \circ d) \circ(\delta c)^{*}-(\delta b \circ d) \circ c^{*}-(b \circ \delta d) \circ c^{*} \\
& +\left(c^{*} \circ d\right) \circ \delta b-\left((\delta c)^{*} \circ d\right) \circ b+\left(c^{*} \circ \delta d\right) \circ b \\
= & \left\{\delta b, c^{*}, d\right\}-\left\{b,(\delta c)^{*}, d\right\}+\left\{b, c^{*}, \delta d\right\} .
\end{aligned}
$$

Corollary 10. If $\delta$ is $a{ }^{*}$-derivation on a $J B^{*}$-algebra $A$, then $\delta \in \operatorname{Her} B(A)$.

Proof. $\exp (i t \delta)$ is a ${ }^{*}$-automorphism for all $t \in \mathrm{R}$, and hence is an isometry by (13) Corollary 1.4. Thus $\| \exp$ it $\delta \|=1$ for all $t \in R$, and so $\delta \in \operatorname{Her} B(A)$ by (4) Lemma 5.2.

We now come to the analogue of Sinclair's results on Hermitian operators on $C^{*}$-algebras.

Theorem 11. If $A$ is a $J B^{*}$-algebra, and $\lambda \in \operatorname{Her} B(A)$, then $\lambda=L_{a}+\delta$ where $a \in \operatorname{Her}(A)$ and $\delta$ is $a^{*}$-derivation on $A$. Moreover, this decomposition is unique.

Proof. Let $a=\lambda(1)$. Given $f \in D(A, 1)$, define $F \in(B(A))^{\prime}$ by

$$
F(\sigma)=f(\sigma(1))
$$

for $\sigma \in B(A)$. As $F(1)=1$ and $|F(\sigma)| \leqslant\|\sigma(1)\| \leqslant\|\sigma\|$, we have $F \in D(B(A), 1)$. Hence $f(a)=f(\lambda(1))=F(\lambda) \in R$, and so $a \in \operatorname{Her} A$. By Theorem 6, $L_{a} \in \operatorname{Her} B(A)$, and so by (4) Lemma 5.4, $\delta=\lambda-L_{a} \in \operatorname{Her} B(A)$. As $\delta(1)=0$, $(\exp (i t \delta))(1)=1$ for all $t \in R$, and by (4) Lemma 5.2, as $\delta \in \operatorname{Her} B(A), \exp (i t \delta)$ is an isometry of $A$ onto itself, for all $t \in \mathbb{R}$. By (14) Theorem $6, \exp (i t \delta)$ is thus a *automorphism of $A$ for all $t \in \mathbb{R}$, and so by Theorem 9(iii), $\delta$ is a ${ }^{*}$-derivation.

Finally, as every derivation maps the unit of $A$ to zero, it follows that the decomposition of $\lambda$ is unique.

We use Theorem 11 to derive an algebraic characterisation of Hermitian operators on $J B^{*}$-algebras, which was also obtained by Kaup (8).

Theorem 12. Let $A$ be a $J B^{*}$-algebra, and $\lambda \in B(A)$. Then $\lambda \in \operatorname{Her} B(A)$ if and only if, for all $b, c$ and $d$ in $A$,

$$
\lambda\left\{b, c^{*}, d\right\}=\left\{\lambda b, c^{*}, d\right\}-\left\{b,(\lambda c)^{*}, d\right\}+\left\{b, c^{*}, \lambda d\right\} .
$$

Proof. If $\lambda \in \operatorname{Her} B(A)$, then by Theorem $11, \lambda=L_{a}+\delta$ where $a \in \operatorname{Her} A$ and $\delta$ is a *-derivation. It is sufficient to show that (4) holds for $L_{a}$ and for $\delta$. From (6) page 37, for all $b, c$ and $d$ in $A$,

$$
\begin{aligned}
L_{a}\left\{b, c^{*}, d\right\} & =\left\{L_{a} b, c^{*}, d\right\}-\left\{b, L_{a}\left(c^{*}\right), d\right\}+\left\{b, c^{*}, L_{a} d\right\} \\
& =\left\{L_{a} b, c^{*}, d\right\}-\left\{b,\left(L_{a}(c)\right)^{*}, d\right\}+\left\{b, c^{*}, L_{a} d\right\}
\end{aligned}
$$

as $a$ is self-adjoint. So $L_{a}$ satisfies (4), while $\delta$ satisfies (4) by Theorem 9(iv).

Conversely suppose $\lambda \in B(A)$ satisfies (4), and let $a=\lambda(1)$. Choosing $b=c=d=$ 1 , we have

$$
\lambda(1)=\lambda(1)-(\lambda(1))^{*}+\lambda(1) \text {. }
$$


so $a=\lambda(1)$ is self-adjoint. By Theorem $6, L_{a} \in \operatorname{Her} B(A)$. Let $\psi=\lambda-L_{a}$. Then $\psi(1)=0$ and, by the first part of the proof, $\psi$ also satisfies (4). Taking $d=1$, for all $b$ and $c$ in $A$, it follows that

$$
\psi\left(b \circ c^{*}\right)=\psi(b) \circ c^{*}-b \circ(\psi(c))^{*} .
$$

Choosing $b=1$, for all $c$ in $A, \psi\left(c^{*}\right)=-(\psi(c))^{*}$, and so, for all $b$ and $c$ in $A$,

$$
\psi(b \circ c)=\psi(b) \circ c+b \circ \psi(c) \text {. }
$$

Thus $\psi$ is a ${ }^{*}$-derivation, and so $\lambda=L_{a}+\psi \in \operatorname{Her} B(A)$ by Theorem 11 .

By linearisation, we may replace (4) by apparently weaker hypothesis: we shall only prove part of the following Corollary as an example of the technique.

Corollary 13. Let $A$ be a $J B^{*}$-algebra, and $\lambda \in B(A)$. Then the following are equivalent:

(i) $\lambda \in \operatorname{Her} B(A)$,

(ii) $\lambda\left\{a, b^{*}, a\right\}=2\left\{\lambda a, b^{*}, a\right\}-\left\{a,(\lambda b)^{*}, a\right\}$ for all $a$ and $b$ in $A$,

(iii) $\lambda\left\{a, a^{*}, a\right\}=2\left\{\lambda a, a^{*}, a\right\}-\left\{a,(\lambda a)^{*}, a\right\}$ for all $a$ in $A$.

Proof. From Theorem 12, (i) implies (ii). (ii) implies (iii) is trivial. We shall prove (ii) implies (i), the remaining implication being similar.

Hence, suppose (ii) holds. As for all $a, b$ and $c$ in $A$,

$$
\begin{aligned}
2\left\{a, b^{*}, c\right\}= & \left\{a+c, b^{*}, a+c\right\}-\left\{a, b^{*}, a\right\}-\left\{c, b^{*}, c\right\}, \\
2 \lambda\left\{a, b^{*}, c\right\}= & 2\left\{\lambda(a+c), b^{*}, a+c\right\}-\left\{a+c,(\lambda b)^{*}, a+c\right\}-2\left\{\lambda a, b^{*}, a\right\} \\
& +\left\{a,(\lambda b)^{*}, a\right\}-2\left\{\lambda c, b^{*}, c\right\}+\left\{c,(\lambda b)^{*}, c\right\} \\
= & 2\left\{\lambda c, b^{*}, a\right\}+2\left\{\lambda a, b^{*}, c\right\}-2\left\{a,(\lambda b)^{*}, c\right\} \\
= & 2\left(\left\{a, b^{*}, \lambda c\right\}+\left\{\lambda a, b^{*}, c\right\}+\left\{a,(\lambda b)^{*}, c\right\}\right),
\end{aligned}
$$

for all $a, b$ and $c$ in $A$. Hence by Theorem 12, $\lambda \in \operatorname{Her} B(A)$.

As an application of Theorem 12, we characterise those $a \in \mathrm{Her} A$ such that $\left(L_{a}\right)^{2} \in \operatorname{Her} B(A)$.

Theorem 14. Let $A$ be a $J B^{*}$-algebra, and $a \in \operatorname{Her} A$. Then $\left(L_{a}\right)^{2} \in \operatorname{Her} B(A)$ if and only if $a$ is in the centre of $A$.

Proof. As $\left(L_{a}\right)^{2}=\frac{1}{2}\left(U_{a}+L_{a^{2}}\right)$, we have $\left(L_{a}\right)^{2} \in \operatorname{Her} B(A)$ if and only if $U_{a} \in$ Her $B(A)$. Suppose first that $U_{a} \in \operatorname{Her} B(A)$. Let $h \in \operatorname{Her} A$. By Corollary $4, h=$ $k^{2}-l^{2}$ where $k$ and $l$ are positive elements of the $B^{*}$-algebra which is the closure of the complex Jordan algebra generated by $l$ and $h$. Then putting $c=b=k$ and $d=1$ in (4), we have

$$
U_{a}\{k, k, 1\}=\left\{U_{a}(k), k, 1\right\}-\left\{k,\left(U_{a}(k)\right)^{*}, 1\right\}+\left\{k, k, U_{a}(1)\right\} .
$$

As $\left(U_{a}(k)\right)^{*}=U_{a}(k)$, since $a, k$ are self-adjoint, this means

$$
\left\{a, k^{2}, a\right\}=k^{2} \circ a^{2} \text {. }
$$


Similarly $\left\{a, l^{2}, a\right\}=l^{2} \circ a^{2}$, so that

$$
\{a, h, a\}=h \circ a^{2} .
$$

Thus by Theorem 5, $a$ is in the centre of $A$.

Conversely suppose $a$ is in the centre of $A$. Let $b \in \operatorname{Her} A$. As in part (a) of the proof of Theorem $5, a^{2} \circ b=a \circ(a \circ b)$ or

$$
L_{a^{2}}(b)=\left(L_{a}\right)^{2}(b) \text {. }
$$

Hence, $\left(L_{a}\right)^{2} \in \operatorname{Her} B(A)$, as $L_{a^{2}} \in \operatorname{Her} B(A)$.

Acknowledgement. I wish to thank Professor F. F. Bonsall for his help and encouragement, and the Carnegie Trust for a Carnegie Scholarship.

Note added in proof. Since the submission of this manuscript, an alternative proof of Theorem 11 has been obtained using (16), Proposition 5.4 and (17) Theorem 3.3.

\section{REFERENCES}

(1) E. M. Alfsen, F. W. Shultz, and E. Størmer, A Gelfand-Neumark Theorem for Jordan algebras, Advances in Math. 28 (1978), 11-56.

(2) F. F. BONSALL, Jordan algebras spanned by Hermitian elements of a Banach algebra, Math. Proc. Camb. Phil. Soc. 81 (1977), 3-13.

(3) F. F. BONSALl and J. DunCAN, Complete Normed Algebras (Springer-Verlag, Berlin, Heidelberg, New York, 1973).

(4) F. F. BONSALL and J. DUNCAN, Numerical ranges of operators on normed linear spaces and of elements of normed algebras, (London Math. Soc. Lecture Note Series 2, Cambridge University Press, 1971).

(5) H. BRAUN and M. KoEchER, Jordan-Algebren (Springer-Verlag, Berlin, Heidelberg, New York, 1966).

(6) N. JACOBSON, Structure and Representations of Jordan algebras (Amer. Math. Soc. Colloquim Publications 39, Providence, 1968).

(7) P. JoRdAN, J. VON NEUMANN, and E. WigNER, On an algebraic generalisation of the quantum mechanical formulation, Ann. of Math. (2) 35 (1934), 29-64.

(8) W. KAuP, Algebraic Characterisation of Symmetric Complex Banach Manifolds, Math. Ann. 228 (1977), 29-64.

(9) S. SAKAI, $C^{*}$-algebras and $W^{*}$-algebras (Springer-Verlag, Berlin, Heidelberg, New York, 1971).

(10) A. M. SinClAIR, Jordan homomorphisms and derivations of semi-simple Banach algebras, Proc. Amer. Math. Soc. 24 (1970), 209-214.

(11) E. StøRmer, On the Jordan structure of $C^{*}$-algebras, Trans. Amer. Math. Soc. 120 (1965), 438-447.

(12) D. M. TopPING, Jordan Algebras of self-adjoint Operators, Mem. Amer. Math. Soc. 53 (1965), $1-48$.

(13) J. D. M. WRIGHT, Jordan C*-algebras, Mich. Math. J. 24 (1977), 291-302.

(14) J. D. M. WRIGHT, and M. A. Youngson, On Isometries of Jordan algebras, J. London Math. Soc. (2) 17 (1978), 339-344. 
(15) M. A. Youngson, A Vidav Theorem for Banach Jordan Algebras, Math. Proc. Camb. Philos. Soc. 84 (1978) 263-272.

(16) W. KAUP and H. UPMEIER, Jordan Algebras and symmetric Siegel Domains in Banach spaces, Math. Zeit. 157 (1977), 179-200.

(17) R. BRAUN, W. KAUP and H. UPMEIER, A Holomorphic Characterisation of Jordan $C^{*}$-algebras, Math. Zeit. 161 (1978), 277-290.

Mathematics Department,

UNIVERSITY OF EDINBURGH. 